\title{
«¿Mayor, yo? ¿Dónde lo pone?» \\ Concepción y atribuciones sociales a la vejez según la autopercepción de las personas mayores del siglo XXI: de la seneficiencia al elder pride
}

\author{
"Am I an old person? Where does it say that?" \\ Conception and social attributions to old age related to \\ self-perception of the older people in the 21st century: \\ from senefficiency to elder pride
}

TERESA AMEZCUA (Universidad de Jaén) y MARTA GARCÍA DOMINGO ${ }^{1}$ (Universidad de Jaén)

Artículo recibido: 22 de febrero de 2021

Solicitud de revisión: 2 de abril de 2021

Artículo aceptado: 5 de noviembre de 2021

Amezcua, Teresa y García Domingo, Marta (2022). « ¿Mayor, yo? ¿Dónde lo pone?» Concepción y atribuciones sociales a la vejez según la autopercepción de las personas mayores del siglo XXI: de la seneficiencia al elder pride. Recerca. Revista de Pensament $i$ Análisi, ${ }_{27}$ (1), pp. 1-28. doi: http://dx.doi.org/10.6035/recerca.5778

Resumen

Las personas mayores, como grupo poblacional, son objeto de estereotipos y prejuicios hacia la vejez que derivan en situaciones de discriminación y edadismo. El presente estudio cualitativo indaga en la concepción y las atribuciones sociales a la vejez en las sociedades posindustriales, centrándose en el caso de Alemania y España. Para ello, a través de las técnicas de la entrevista y el grupo focal, se recaba la perspectiva tanto de expertos en el tema como de las propias personas mayores. El análisis evidencia la imprecisión del concepto vejez y las connotaciones negativas asociadas al mismo, así como un rechazo a autoidentificarse como persona mayor. Se concluye la necesidad de una reconceptualización de la vejez que refleje su significado actual.

Palabras clave: personas mayores, conceptualización, atribuciones sociales, estereotipos, edadismo.

\footnotetext{
${ }^{1}$ mgdoming@ujaen.es
} 


\begin{abstract}
Older people, as a population group, are subject to stereotypes and prejudices towards old age that lead to situations of discrimination and ageism. This qualitative study investigates the conception and social attributions of old age in post-industrial societies, focusing on the case of Germany and Spain. For this purpose, through the techniques of the interview and the focus group, the perspective of both experts in the field and elderly themselves is collected. The analysis shows the imprecision of old age concept and the negative connotations associated with it, as well as a refusal to self-identify as an elderly person. The need for a reconceptualization of old age that reflects its current meaning is concluded.
\end{abstract}

Key Words: older people, conceptualization, social attributions, stereotypes, ageism.

\title{
INTRODUCCIÓN
}

Asistimos a un cambio sociodemográfico radical, caracterizado por un notorio envejecimiento poblacional y la prolongación de la etapa tradicionalmente concebida como vejez. En este escenario, analizar la realidad de los mayores, las atribuciones sociales que condicionan su vivencia de la vejez y las emociones que experimentan es una responsabilidad ineludible para las ciencias sociales y humanísticas (Amezcua y García-Domingo, 2020). Sin embargo, la incorporación de la experiencia emocional de la vejez en la disciplina científica es aún incipiente (Márquez-González, 2008).

En este sentido, este estudio pretende avanzar en la clarificación del concepto persona mayor en las sociedades posindustriales, así como analizar las atribuciones sociales asignadas a las personas mayores como individuos y como colectivo, tomando como punto de partida su propia vivencia.

\section{EVOLUCIÓN DE LA CONCEPCIÓN SOCIAL DE LA VEJEZ}

El curso vital se estructura tradicionalmente en tres etapas asociadas a la edad: aprendizaje-niñez, trabajo-adultez y retiro-vejez, cuya duración se define según los modos de vida propios de cada sociedad. Esta compartimentación es una herramienta de control social que favorece la reproducción de desigualdades sociales (García y Alfageme, 2012), especialmente durante la vejez.

En esta línea, el debate en torno a la vejez discurre entre dos aproximaciones teóricas divergentes, que la clasifican también en varias etapas en función de los elementos sobre los que se hace énfasis. Por un lado, los modelos feno- 
menológicos (Neugarten, 1974), centrados en describir las características del envejecimiento saludable y activo, subrayan la dicotomía joven-viejo (Johnson y Barer, 1997), incidiendo en el estereotipo negativo de la vejez y focalizando su atención en la enfermedad y el deterioro (Rowe y Khan, 1997). Por su parte, los modelos procesuales como el structural lag (Riley y Riley, 1994), el coping proactivo (Kahana y Kahana, 1996) o el envejecimiento positivo (FernándezBallesteros, Schettini, Santacreu y Molina, 2012) estudian la vejez como constructo social, contemplando la incidencia de los factores sociales y estructurales en el proceso de envejecimiento multidimensional. Fomenta un estereotipo positivo de vejez, destacando el envejecimiento poblacional como un logro del desarrollo económico y social y de salud pública (Fries, 1980), subrayando la integración, la contribución social y la satisfacción vital de las personas mayores.

Desde este paradigma, la vejez es un proceso biológico, psicológico y social que implica y requiere cambios adaptativos a nivel cognitivo, emocional y funcional. Precisamente, el modo de envejecer se ve influenciado por aspectos socioculturales vinculados con la organización social para la satisfacción de necesidades que proporcionarán tanto opciones como restricciones. Así, cada cultura y cada periodo histórico definen un esquema adecuado de curso vital, con patrones comportamentales delimitados para cada etapa «a menudo falsos y artificiales» (Gutiérrez y Hernández, 2013: 139). No obstante, estudios previos evidencian que, independientemente del contexto cultural, el buen envejecer es definido por los mayores en relación con: tener buena salud, estar satisfecho con la vida, tener amigos y familiares, adaptarse a los cambios y cuidar de uno mismo (Fernández-Ballesteros, Schettini, Santacreu y Molina, 2012)

Al igual que otros estatus sociales adscritos, como el género, la clase o la etnia, la edad es un elemento de identidad social (Barrett, Redmond y Von Rohr, 2012). Pizzorno (1978) define la identidad colectiva como un constructo subjetivo y cambiante articulado a partir de la existencia de una comunidad imaginada, la pertenencia a un grupo como círculo de reconocimiento y la ejecución de rituales de reafirmación de la acción colectiva. A su vez, dicho elemento identitario se ve determinado por otros estatus sociales cuyo análisis merece especial atención a través de estudios específicos y en profundidad.

A ello hay que añadir que la identidad individual depende, en gran medi$\mathrm{da}$, de la función desarrollada o asignada. Es decir, somos o valemos en tanto tenemos un rol que representar y producimos unos beneficios sociales. La edad social define los roles socioculturalmente atribuidos a una etapa determinada del ciclo vital y que se vinculan a las expectativas sobre su contribución a la 
comunidad. Así, al dejar de ocupar un rol laboral económicamente productivo, desde la óptica de la sociedad capitalista las personas mayores pierden su función social. En la cultura mediterránea, las personas ancianas disfrutaban de un estatus preeminente como sabios, atesorando y transmitiendo oralmente el conocimiento colectivo. Consecuentemente, con la institucionalización de la educación reglada perdieron parte de su función social, situación intensificada en las sociedades posindustriales (Amezcua y Alberich, 2017) provocando el desempeño de lo que Clavan (1978) denomina un «rol sin rol».

Desde este paradigma surgen las categorizaciones de tercera y cuarta edad como etapas de la vejez. La tercera edad se fija a partir de los 65 años independientemente de la situación personal, actividad profesional o estado de salud (De Jouvenel, 1989). En esta etapa, las mejoras de las condiciones de vida en las sociedades avanzadas proporcionan oportunidades para realizar múltiples actividades durante la jubilación, pudiéndose convertir en una etapa vital altamente gratificante (Riley y Riley, 1994). En esta fase del curso vital, se recupera un papel protagonista y un espacio público para las personas mayores desde el enfoque del envejecimiento exitoso, que dista de otros enfoques como el envejecimiento activo o el saludable al encontrar su fundamento en el bienestar subjetivo. Este, a su vez, es concebido como el grado de satisfacción experimentado por las personas al hacer un juicio global de su trayectoria vital (García-Rodríguez, 2007)

Sin embargo, el envejecimiento del envejecimiento o cuarta edad, cuyo inicio se establece entre los 8 o u 85 años, conlleva un progresivo deterioro físico, psicológico y social, trazando una división cualitativa entre los que son y los que todavía no son realmente mayores (Higgs y Gilleard, 2021). Se ha demostrado en estudios previos (Katz, Holland y Peace, 2013) que las variables biológicas, psicológicas y sociales se interrelacionan y afectan al bienestar objetivo de los individuos, produciendo pérdida de autonomía y situaciones de dependencia.

En una línea similar, pero incidiendo en la disminución de contribución social, la teoría de «las tres etapas de la jubilación» (Reitzes y Mutran, 2004) categoriza a las personas mayores en función de su nivel de actividad. La idea central es que, tras la jubilación, hay tres periodos de adaptación al nuevo estatus. En la primera etapa, las personas mayores, denominadas Go-Go, mantienen un alto grado de actividad, competencia y autonomía. En la segunda etapa, la de los Slow-Go, comienzan a sufrir problemas de movilidad y accesibilidad que limitan su actividad. En la última etapa, los mayores son ya dependientes, son los No-Go. 
Vinculado a estas clasificaciones, el estatus de las personas mayores es actualmente inestable, pues depende de la combinación de un conjunto de variables - personales y psicológicas (autoconcepto, identidad individual y orientación sexual), sociales (de género, de clase, raciales y étnicas), de salud (dicotomía salud-enfermedad, discapacidad), económicas, etc.- que se producen en diferentes grados y que ubican al individuo en un grupo o subgrupo poblacional. Debido a esta heterogeneidad, al analizar la situación de las personas mayores es necesario tener en cuenta no solo su edad, sino también factores físico-orgánicos, psicológicos, educativos, culturales, sociales y familiares, la intensidad de las relaciones sociales, la clase social y el nivel de ingresos.

\section{AUSENCIA DE RECONOCIMIENTO Y DISCRIMINACIÓN DE LA VEJEZ EN LAS SOCIEDADES POSINDUSTRIALES}

Una vez evidenciada la relevancia de los valores culturales y las representaciones colectivas como elementos de construcción de la identidad social, cabe reflejar la ausencia de reconocimiento a la vejez propia de las sociedades posindustriales. Surgen así nuevas realidades como el edadismo, definido por Butler (1975: 12) como «un proceso de estereotipos y discriminación sistemáticos contra las personas mayores porque son viejas». Dos elementos clave respaldan el edadismo: de un lado, el imaginario social de la vejez como declive o pérdida; de otro, los discursos homogeneizadores de la vejez (Medina, 2018; Sandberg, 2013). La discriminación, propia del edadismo, se asocia y encuentra su raíz en la extensión de estereotipos y prejuicios hacia la vejez, si bien se distancia de estos en que, en el caso de la primera, las conductas discriminatorias no son encubiertas, sino observables (Ribera et al., 2016). Según Amico (2009), las actitudes y las conductas discriminatorias encontrarían su base en la ausencia de reconocimiento y la valoración negativa estereotipada hacia las personas mayores, dando lugar a situaciones de aislamiento, maltrato, institucionalización, trato impersonal o falta de respeto, entre otras. A su vez, esta valoración negativa estereotipada parte de heurísticos con límites evidentes, como la merma de capacidades y el envejecimiento (Cook y Halsall, 2012), que obvia cuestiones vinculadas a la concepción, la percepción social y el trato directo a las personas mayores y las implicaciones que conllevan dichos factores. Nos encontramos, por tanto, con una dicotomía entre lo socialmente deseable frente a la cotidianidad en las actitudes y trato hacia las personas mayores. A tal efecto, mientras prevalece un discurso sobre la deseabilidad y 
pertinencia de desarrollar políticas sociales que generen oportunidades de participación para los mayores y los empoderen como colectivo, nos encontramos con prácticas discriminatorias manifiestas y observables (Ribera et al., 2016), que van desde el paternalismo a conductas evitativas.

\section{OBJETIVOS Y METODOLOGÍA}

La presente investigación se plantea como objetivo general indagar en el imaginario social de la vejez en las sociedades posindustriales, a partir del caso de Alemania y España. Se seleccionan estos contextos por representar distintos modelos de estado de bienestar: el corporativista y el mediterráneo. El estudio pretende avanzar en el conocimiento de la conceptualización de la vejez, las atribuciones sociales hacia esta y su impacto en el bienestar percibido por los propios mayores. El objeto de estudio es abordado desde un enfoque evolutivo y tendencial.

Para comprender en profundidad esta realidad se utiliza una metodología cualitativa basada en las técnicas de la entrevista en profundidad y el grupo focal (en adelante, GF). Cada técnica se emplea con uno de los dos grupos de interés - expertos y personas mayores-, cuya participación se considera clave para lograr la representatividad de los discursos y la complementariedad de la información recabada. En concreto, se realizan 33 entrevistas semiestructuradas -22 a expertos y 11 a mayores líderes comunitarios-, precedidas por tres de validación; y dos GF con un total de 17 personas mayores. La muestra final estuvo compuesta por 50 participantes y determinada por la saturación de los discursos. Las personas expertas entrevistadas representan dos perfiles profesionales considerados relevantes para el análisis propuesto: académicos/as e investigadores/as de reconocido prestigio en la materia - perfil I del grupo de expertos-; y profesionales de campo con personas mayores - perfil II del grupo de expertos. Entre los criterios de inclusión se garantizó que las distintas disciplinas sociales estuvieran representadas y que contaran con una trayectoria profesional mínima de 5 años en el ámbito y el contexto objeto de estudio. Por su parte, las percepciones y las subjetividades del colectivo de personas mayores (recogidas a través de participantes entre 6o y 87 años) fueron recogidas a partir de un perfil doble y el empleo de dos técnicas. En concreto, entrevistamos en profundidad a mayores que actúan como líderes en su comunidad - perfil I del grupo de mayores-, complementando esta información con la que nos aportan los mayores que, sin ser líderes comunitarios, participan en 
alguna entidad asociativa - perfil II del grupo de mayores- y que desarrollan sus discursos a partir del empleo de la técnica del grupo focal. Entre los criterios de inclusión, se consideró la edad -6o años o más- y la participación en una entidad asociativa. En lo relativo a los contextos de interés -zona I. Alemania y zona II. España-, ambos tuvieron una representación equitativa -23 informantes de la zona I y 27 de la zona II. Para garantizar la representatividad de los discursos de distintos grupos de mayores, se incluyó la variable de clase social en la selección de informantes. Esta se determina en función del nivel educativo - estudios primarios, secundarios y superiores - y de ingresos - con base en el porcentaje de salario mínimo interprofesional—, recogiéndose así el discurso de mayores integrados en la categoría de precariado, clase media y clase alta, si bien hay una mayor representación de la clase media en la muestra final.

Tabla 1. Cuadro descriptivo del trabajo de campo y perfil de los participantes

\begin{tabular}{|c|c|c|c|c|c|c|c|c|c|c|c|c|c|c|}
\hline \multicolumn{4}{|c|}{ Perfil } & \multicolumn{2}{|c|}{ Edad } & \multicolumn{2}{|c|}{ Sexo } & \multicolumn{3}{|c|}{ Clase social } & \multicolumn{2}{|c|}{ Zona } & \multicolumn{2}{|c|}{ Técnica } \\
\hline \multicolumn{2}{|c|}{ Experto } & \multicolumn{2}{|c|}{ Persona Mayor } & \multirow{2}{*}{$\begin{array}{c}3 . a^{\prime} \\
\text { edad }\end{array}$} & \multirow{2}{*}{$\begin{array}{r}4,{ }^{a} \\
\text { edad } \\
\end{array}$} & \multirow[t]{2}{*}{ Mujer } & \multirow[t]{2}{*}{ Hombre } & \multirow[t]{2}{*}{ Precariado } & \multirow[t]{2}{*}{ Media } & \multirow[t]{2}{*}{ Oligarquía } & \multirow[t]{2}{*}{ Alemania } & \multirow[t]{2}{*}{ España } & \multirow[t]{2}{*}{ Entrevista } & \multirow{2}{*}{$\begin{array}{l}\text { Grupo } \\
\text { focal }\end{array}$} \\
\hline Académico & Profesional & Lider & Socio/a & & & & & & & & & & & \\
\hline 9 & 13 & 11 & 17 & 25 & 3 & 15 & 13 & 6 & 18 & 4 & 23 & 27 & 33 & 17 \\
\hline \multicolumn{2}{|c|}{22} & \multicolumn{2}{|c|}{28} & & & & & & & & & & & \\
\hline \multicolumn{4}{|c|}{50} & \multicolumn{2}{|c|}{28} & \multicolumn{2}{|c|}{28} & \multicolumn{3}{|c|}{28} & \multicolumn{2}{|c|}{50} & \multicolumn{2}{|c|}{50} \\
\hline
\end{tabular}

Fuente: elaboración propia

En el desarrollo de las dos técnicas se siguió un guion semiestructurado con preguntas abiertas, siendo estas grabadas e íntegramente transcritas. En el proceso de análisis se empleó el software Atlas.ti-V8 para la codificación, categorización y sistematización del enraizamiento (E) y densidad (D) de los resultados y la identificación de redes conceptuales.

Para garantizar el anonimato de los participantes, se les asigna un código alfanumérico con información relevante sobre su perfil, pero que no permite su identificación.

Tabla 2

Códigos identificativos de los participantes

\begin{tabular}{|l|l|l|}
\hline \multirow{2}{*}{ Contexto } & Alemania & A \\
\cline { 2 - 3 } & España & E \\
\hline \multirow{2}{*}{ Perfil } & Persona mayor & $\mathrm{m}$ \\
\cline { 2 - 3 } & Experto & $\mathrm{e}$ \\
\hline
\end{tabular}




\begin{tabular}{|l|l|l|}
\hline \multirow{3}{*}{ Clase social* $^{*}$} & Oligarquía & $\uparrow$ \\
\cline { 2 - 3 } & Clase media & $\leftrightarrow$ \\
\cline { 2 - 3 } & Precariado & $\downarrow$ \\
\hline Edad* $^{*}$ & $60-89$ & $\mathrm{~N}$ \\
\hline \multirow{2}{*}{ Sexo* } & Masculino & $\sigma^{\top}$ \\
\cline { 2 - 3 } & Femenino & ○ \\
\hline Participante & $1-50$ & $\mathrm{~N}$ \\
\hline
\end{tabular}

*Solo se especifica en el caso de los participantes con perfil personas mayores Fuente: elaboración propia

\section{LOS LÍMITES DIFUSOS EN LA CONCEPTUALIZACIÓN DE LA VEJEZ}

Como se ha mencionado, la condición de vejez reúne una serie de condicionantes personales, psicológicos, físicos, sociales y económicos. El resultado de la combinación de esos factores produce una conceptualización de vejez distinta para cada individuo.

Ser mayor es diferente en cada persona, son muchos los factores, cada persona es un mundo en la vejez (Ae17)

La multiplicidad de factores susceptibles de incidir en la situación de vejez produce confusión entre gran parte de los participantes en los GF, dificultando la conceptualización de la vejez. Al ser preguntados «¿cuándo es una persona mayor?», requieren de unos segundos para recapacitar sobre ello, e incluso modifican el discurso mientras este se desarrolla. Si bien normativamente el indicador de vejez es la edad cronológica (65 años o más), la significación actualmente atribuida al concepto es difusa, existiendo diferentes discursos en función de qué factores del envejecimiento sean considerados. No obstante, prevalece la distinción entre los dos elementos señalados por De Jouvenel (1989): la situación laboral y el estado de salud.

Ser mayor es cuando ya no puedes hacer cosas... o bueno, cuando ya no tienes ganas de hacerlas, porque a veces siendo joven tampoco... no sé. (Em $\uparrow 74{ }_{40}$ )

Cuando te jubilas eres mayor. (...) en realidad da igual, lo que importa es que tengas salud. Si uno está bien, dan igual los años $\left(\mathrm{Em} \leftrightarrow 800^{3} 6\right)$ 
Incluso en línea con otros estudios (Cantos, 2019), la terminología social y científica vigente parece ser ajena a la construcción de la conceptualización del fenómeno por parte de las propias personas mayores, que manifiestan desconocimiento - $\mathrm{e}$ incluso desconcierto- ante términos como tercera y cuarta edad, edadismo o gerontofobia, vinculadas estrechamente a las actuaciones sociopolíticas gerontológicas.

¿Cuarta edad? ¿Ya vamos por ahí? (risas) Pues estaba yo mejor en la primera o la segunda $\left(\mathrm{Em} \leftrightarrow 730^{3}\right)$

En este sentido, las personas informantes expertas evidencian que la objetivación normativa de la vejez a los 65 años como frontera entre adultez y vejez es un convencionalismo que responde a razonamientos obsoletos pues «ya no hay un criterio objetivo para estipular qué es ser mayor» (Ee17), respondiendo esto a un interés sociopolítico de establecer un paralelismo entre edad cronológica de vejez y edad de jubilación. Sin embargo, las reformas de los últimos años en las sociedades posindustriales que han retrasado la edad de jubilación hasta los 67 años rompen la analogía entre retiro laboral y senectud, dificultando identificar cuándo se es una persona mayor.

Es que ahora el problema de la vejez es en qué edad la fechamos. Que antes, cuando uno se jubilaba, a partir de que se jubilaba ya pasaba a la tercera edad. Y hoy no, hoy con 65 años... (Ee22)

También se apunta la inexistencia de unificación de criterios para todas las áreas de aplicación en el diseño de políticas sociales para personas mayores, estableciéndose dispares franjas de edad al catalogarlas. Así, coexisten programas para mayores de 55 años que promueven el envejecimiento activo desde el área de la salud o la educación, como las aulas universitarias, y otros, a partir de los 6o, como el Programa de Turismo Social.

Los nuevos mayores que están llegando ahora mismo no se consideran mayores. Llegan con unas necesidades vitales que no tienen que ver nada con las de hace veinte años, o treinta, que es cuando se diseñaron ciertos tipos de estrategias y recursos para los mayores $\left(\mathrm{Ee}_{5}\right)$

Cuando tenía 55 años me llamaron a un curso de mujeres mayores de informática en la oficina de voluntariado que te dije, y fui a allí y le dije al hombre: «¿Perdone? ¡Esto no está bien!». Y él me dijo: «Bueno, estás en la lista en el ordenador y la invitación se envía 
por edades y encajas en esta franja» (risas). Entonces, esa fue la primera vez que me di cuenta que era una mujer mayor (risas). (Am $\leftrightarrow 63{ }^{\circ} 30$ )

Tal diversidad se ve reflejada en el discurso. Por ejemplo, la identificación entre jubilación y vejez no se realiza de forma automática, al encontrarse realidades sociales disímiles como el aumento de la edad de jubilación o las jubilaciones anticipadas, que posicionan en situación de retiro laboral a edades más tempranas.

A partir de la jubilación..., no se... ¡Hombre! hay quien se prejubila a los 60 o los 62» $\left(\mathrm{Em} \leftrightarrow 74{ }^{\circ} 26\right)$

En este sentido, la jubilación como rito de paso pierde vigencia en la identificación social de la vejez.

¿Por qué hemos decidido que sea el de 65 ? Porque ya no hay un criterio objetivo para que sea de 65, ¿no? Tendríamos que decir mayores de 67, que es la edad de jubilación. Quiero decir que cuando hablamos de esas cosas tenemos que tener un poco de cuidado, de qué estamos hablando (Ee5)

Además del estatus laboral, otros condicionantes modifican las percepciones sobre la vejez. La mejora de las condiciones de vida y los niveles de salud contribuyen a dificultar la identificación del comienzo de la vejez. Justamente, el discurso mayoritario relaciona vejez con malas condiciones de salud, discapacidad y dependencia.

A mí la vejez no me preocupa, me preocupa más la enfermedad (Em $\left.\uparrow 80{ }_{32}\right)$

A veces encuentro personas con 85 años que están fenomenal, con mucha movilidad, y otras personas con 65 que están muy mal. También hay gente menor de 65 años con muchas enfermedades y problemas de salud (Ae17)

La superación de la dicotomía jubilación-vejez parece haberse producido. En este sentido, la línea argumental de los/as expertos/as se debate entre las normas legales y sociales y la reflexión sobre del discurso de los mayores que no se reconocen como tales. 


\section{5. «YO NO SOY MAYOR»: PERCEPCIÓN DE LA VEJEZ SEGÚN LAS PERSONAS MAYORES}

Esta conceptualización de la vejez vinculada a los niveles de salud produce discrepancias entre la asignación socionormativa de la vejez con la edad cronológica y la autoidentificación como persona mayor.

Yo no lo soy. Yo, qué quieres que te diga, yo no me considero una persona mayor (Em $\leftrightarrow$ 67031)

El análisis a nivel lingüístico revela que las personas mayores no se refieren generalmente a sí mismas como tales. Al responder a cuestiones generales sobre la vejez, lo suelen hacer en tercera persona, excluyéndose de la pertenencia al grupo poblacional de personas mayores:

Quizás sea que no estamos acostumbrados a la gente mayor y ellos sí lo están. Ellos, como mayores que son, han vivido otra época en la que se les daba valor (Em $\downarrow$ 66038)

El análisis en el plano semántico denota una contradicción entre la norma social y las expectativas individuales. La convención que sitúa la vejez a partir de 65 años disiente con las autopercepciones que estos tienen de sí mismos, evidenciándose una resistencia a la autoidentificación como viejo.

Yo me considero mayor por los años, pero luego no soy mayor $\left(\mathrm{Em} \leftrightarrow 7_{6927}\right)$

La conceptualización actual de vejez estereotipa a las personas mayores poniendo el foco únicamente en las problemáticas tradicionalmente asociadas a esta, como el deterioro, la enfermedad, la inactividad o la improductividad, dejando de lado otras dimensiones vitales positivas que pudieran definirla.

A las personas mayores las relacionas con que van para abajo, que están en declive (Ae7)

Crees que no va a llegar la edad esta, y no quieres, no te apetece, crees que no vas a hacer nada $(\mathrm{Em} \downarrow 66038)$

Por ello, los entrevistados encuentran dificultades para reconocerse en la idea arquetípica de persona mayor, percibiendo que esta no se corresponde con sus propias vivencias. 
Si te digo cuántos años tengo y me oigo a mí mismo, ¡es terrible! Pero uno se siente joven. Sigmund Freud decía: el alma no envejece, los sentimientos no envejecen. Ese es el problema $\left(\mathrm{Am} \leftrightarrow 680^{2} 29\right)$

En este sentido, nuestros hallazgos contradicen a Barrett, Redmond y Von Rohr (2012) evidenciando que la edad, en el caso de la vejez, no se constituye en elemento de identidad social. Además, los estereotipos y los prejuicios sobre la vejez impiden que esta sea percibida como comunidad imaginada deseable en los términos expuestos por Pizzorno (1978), pues, si solo posee connotaciones negativas, no existen incentivos para la identificación colectiva.

A este respecto, coincidiendo con autores como Andrews (1999) o Sandberg (2013), abogamos por una forma diferente de pensar y aprender sobre el envejecimiento, que vaya más allá de la distinción entre nosotros y ellos y lleve al reconocimiento de nosotros mismos en cada persona mayor (De Beauvoir, 1970).

\section{Figura 1}

Definición confusa de la vejez

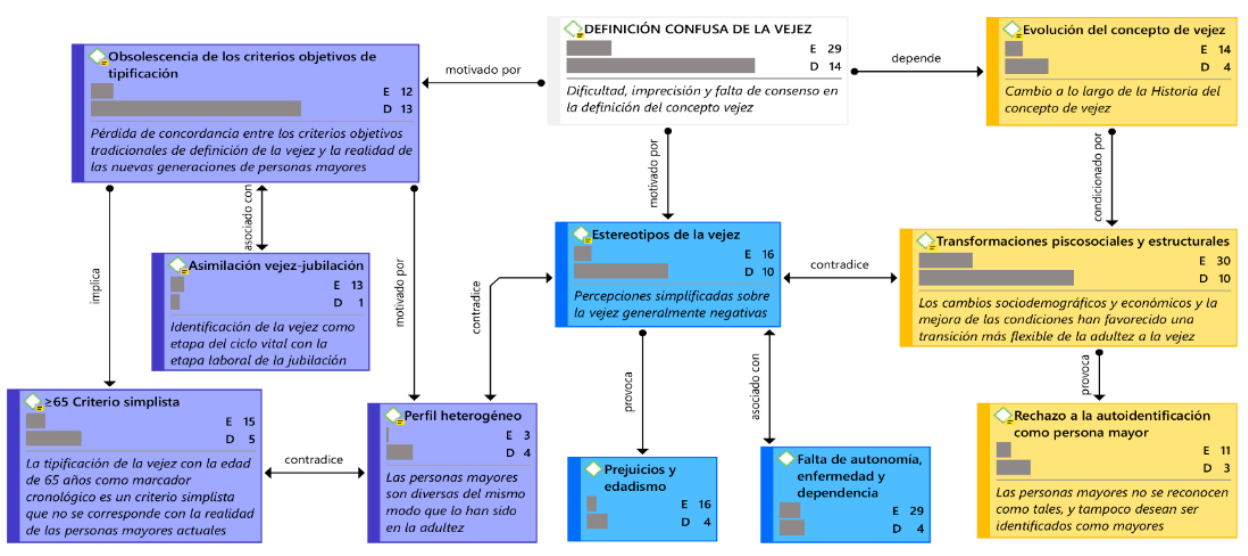

Fuente: elaboración propia

\section{APROXIMACIÓN AL IMAGINARIO SOCIAL DE LA VEJEZ A PARTIR DE LOS DISCURSOS: LA VEJEZ COMO SABIDURÍA, FEALDAD Y CARGA SOCIAL}

El discurso de la vejez se construye a partir de los procesos de socialización en las redes familiares, de amistad, vecindad y comunidad, laborales y las 
redes de los medios de comunicación (Fernández y Kehl, 2001). Este discurso se concreta en normas sociales, culturales y jurídicas que construyen el marco de referencia para la atribución de roles en la vejez. En definitiva, el contexto y la cultura en la que se desarrolla la trayectoria vital de cada individuo predisponen su percepción ante la propia vejez. Como señala Gullette (1997), es la cultura, y no la edad, la que nos envejece. También, cómo percibe el conjunto de la sociedad a un colectivo nos proporciona información sobre el estatus que estructuralmente se le adjudica.

Depende de cada uno como lo ve, en las experiencias que haya tenido con los viejos. (...) Hay quienes los discriminan y, sobre todo los ignorantes, que no tienen interés en aprender de los otros, pero eso es ya cosa de cada uno (Am $\uparrow$ 810 33)

Se dan, por tanto, condicionantes personales, culturales y sociales —nivel educativo, clase social, raza, género, orientación sexual, identidad sexual, origen étnico, religión, entre otros- que determinan distintas vivencias de la vejez incluso en el mismo contexto. Precisamente, al acometer el análisis sobre la imagen social de la vejez, encontramos varios niveles discursivos.

\subsection{La vejez como sabiduría}

Un primer nivel de discurso define a las personas mayores como poseedoras de la sabiduría de la experiencia que, a priori, les confiere una posición preeminente en la sociedad.

Con sabiduría de la vejez se alude al conjunto de conocimientos derivados de la experiencia vital relevantes para el desarrollo de la sociedad porque pueden «aportar cosas que tú no has vivido» (Ee12). Este discurso enlaza con la idea de que las personas mayores son más resilientes que las generaciones posteriores al haber tenido que afrontar situaciones de extrema gravedad, como los conflictos bélicos. En esta línea, Navarro, Bueno, Buz y Mayoral (2006) evidenciaron que, generalmente, los mayores presentan un alto nivel de eficacia en el manejo de problemas, en contra del estereotipo de declive en su capacidad de regulación emocional. Así, los expertos los señalan como un referente de adaptabilidad al medio en condiciones vitales adversas, resaltando, en comparación, la baja resistencia a la frustración de generaciones más jóvenes.

Las personas mayores han vivido tanto, han sufrido tanto en diferentes situaciones, con tantas dificultades, que nos están dando un ejemplo de eso, de adaptabilidad al medio 
(...). Nos enseñan su empuje, su fuerza, su adaptabilidad y sus formas de afrontar esas situaciones de maneras muy diferentes (Ee11)

Sin embargo, las personas entrevistadas manifiestan la percepción de merma de reconocimiento hacia las personas mayores, ya que la sabiduría de la experiencia vital pierde valor en las sociedades posindustriales que disponen de otros medios para gestionar el conocimiento.

Cuando miramos a otras sociedades, (...) las personas mayores son los sabios, personas especiales que tienen un montón de conocimientos y de habilidades que son importantes para esas sociedades. Si vas, por ejemplo, a un pueblecito en África, la persona más mayor del pueblo está viviendo en el centro del pueblo, dentro de pueblo, y todo el mundo va a pedirle consejo, ayuda, a preguntarle cómo hacer las cosas. Porque ellos han visto todo, ellos tienen un montón de tradiciones y de conocimientos en la cabeza (Aeg)

No, no se les da el mérito que se debe. No es como en otras culturas, como la gitana. La importancia que se le da al patriarca o a la matriarca en la vejez se pondera, aquí no. Aquí se desprecia a los mayores (Ee13)

Esta percepción de minusvaloración social se refleja en los discursos de los mayores, desvelándose una contradicción entre lo que debería ser y lo que realmente es, entre lo que la tradición cultural en la que han crecido les ha enseñado y lo que perciben en su entorno. Las personas mayores pierden el rol tradicional de transmisión de conocimiento quedando, en la línea señalada por Clavan (1978) con un «rol sin rol».

Si bien queda patente la necesidad de superar ciertas barreras de las sociedades posindustriales hacia la vejez, el foco de atención de los mayores se centra en la pertinencia de redefinir su nuevo rol social para adaptarse a los nuevos parámetros derivados de la reformulación de los valores tradicionales familiares y el estatus de las personas mayores en la comunidad.

Los jóvenes de hoy no les dan a los mayores la importancia que tienen. Aparte de que la sociedad ha cambiado muchísimo y no tienen el respeto que le teníamos antiguamente. Una persona mayor para nosotros era... ¡Buah! ¡Y que no la respetases, que ahí estaban tus padres para recordártelo! $\left(\mathrm{Em} \leftrightarrow 670^{3}\right)^{2}$

\subsection{La vejez como fealdad}

En línea con estudios previos (Butler, 1980; Nelson, 2005), nuestros resultados evidencian que los prejuicios y los estereotipos sobre la imagen corporal 
de la vejez provocan edadismo y discriminación hacia las personas mayores. Siguiendo a Esteban (2004: 19), «convertirse en un individuo social implica un determinado aprendizaje corporal». De un lado, en línea con Marshall (2012), el fenómeno de la méconnaissance o falta de reconocimiento de la propia imagen corporal ante el espejo provoca una desconexión entre lo que las personas sienten que son y su propia imagen reflejada, especialmente entre las mujeres. Por otro, la representación social de la vejez como etapa asexuada de declive físico provoca un rechazo vinculado a la no aceptación de los cambios corporales discrepantes con los cánones de belleza vigentes, especialmente si se es mujer, ya que, como apunta Gott al hablar del sexy oldie (2004: 25), «para ser sexualmente atractivo a cualquier edad tienes que ajustarte a una noción juvenil de belleza».

En este sentido, los mayores españoles expresan rechazo en el plano físico y una respuesta visceral ante lo viejo en contraposición a lo bello. La vejez como antítesis de uno de los valores más enaltecidos e idealizados en las sociedades posindustriales, la juventud como lo bello y deseable.

La vejez provoca incluso repulsión y rechazo debido a circunstancias físicas, psicológicas y conductuales asociadas al deterioro y la aparición de síndromes geriátricos como la incontinencia urinaria, la movilidad reducida o las limitaciones comunicativas y cognoscitivas que, en otras etapas como la infancia, son juzgadas con mucha mayor indulgencia, naturalizadas y normalizadas.

Te dicen: «iCon los mocos! ¡Con las babas que se les caen!» (Em $\left.\downarrow 69{ }^{9} 37\right)$

Algunas personas mayores enfrentan estos prejuicios con una actitud de resignación, aceptando e incluso justificando el rechazo. Otras expresan indignación ante la estigmatización de la vejez de las generaciones más jóvenes que, en línea con Amico (2009), está basada en el desconocimiento.

Y ahora parece que las personas mayores.... ¿Allí, ¡Allí vas a coger enfermedades! ¡Y la vejez no es enfermedades que te puedan contagiar! (Em $\left.\downarrow 78{ }^{2}{ }_{23}\right)$

La vinculación entre vejez y fealdad deriva en edadismo que, como señalan Ribera et al. (2016), se traduce en comportamientos empíricamente observables que provocan situaciones de exclusión social llegando incluso a la evitación de compartir espacios físicos. Lo paradójico es que, mientras los mayores alemanes observan esta actitud discriminatoria de forma crítica, en el caso español este discurso es incluso asumido por las propias personas mayo- 
res, refiriéndose a individuos de su misma edad con expresiones de repulsa y menosprecio.

Es duro ver que en los medios de comunicación se le da más valor a la juventud, se les coloca más en primer plano por una cuestión de estética (Am $\uparrow$ 810 33)

¿Yo voy a ir ahí con los abuelos? ¡Ni loca! (Em $\leftrightarrow 74$ \&26)

En contraposición a lo señalado en trabajos previos (FernándezBallesteros, Schettini, Santacreu y Molina, 2012), nuestra investigación evidencia que el estilo de afrontamiento del envejecimiento y el impacto de autorreconocerse como persona mayor se ve afectado no solo por prejuicios y estereotipos generales, sino también por el contexto sociocultural. Por ejemplo, se observa una mejor aceptación y reconocimiento de la vejez en el contexto alemán, siendo más acusada la reproducción de prejuicios en el español. Esto se evidencia no solo en la terminología empleada, sino también en la percepción sobre las instituciones de atención a mayores o el rechazo a ser considerado mayor. Sobre esto, el discurso profesional señala la pertinencia de idear una nueva terminología que refleje con más precisión la vejez actual y permita romper con el entramado de prejuicios y estereotipos entorno al concepto, aproximando las definiciones teóricas a las nuevas realidades sociales. Se apunta al trabajo social como disciplina idónea desde la que generar este corpus lingüístico por su rol mediador, proactivo, de influencia y transformación social (García-Domingo y Sotomayor, 2017) en el marco de la intensificación de las relaciones entre el colectivo profesional y los mayores como potenciales usuarios.

\subsection{La vejez como carga social}

A pesar de que numerosos estudios evidencian la contribución social de las personas mayores, principalmente en prestación de cuidados, estas se perciben como una carga social, entendiendo las circunstancias asociadas a la vejez como gravamen para los intereses del conjunto de la sociedad. Así, tanto desde el punto de vista fisiológico — situaciones de enfermedad y discapacidad-como social — situaciones de dependencia - o económico - gasto social que implica-, las personas mayores son consideradas una carga para la institución social que ha de prestarle apoyo. 
En el caso de España, se evidencia el impacto del modelo de bienestar social mediterráneo, caracterizado por unos gastos asistenciales mínimos (Sapir, 2006) y por un modelo de provisión de bienestar fuertemente familista, en el que la responsabilidad de cuidados de la vejez recae en la familia y especialmente en las mujeres (García-Domingo, 2019). Los mayores españoles se perciben como una carga para sus familias, para las que la norma social de los cuidados obstaculiza el trascurso natural de sus vidas. Los mayores expresan malestar por esta minusvaloración y falta de reconocimiento a su contribución social. Este discurso enlaza con el de la sabiduría, reivindicando su valía como portadores del conocimiento de la experiencia. También se reclama el reconocimiento a su contribución en las tareas de prestación de cuidados de las personas dependientes - tanto mayores como menores- en el ámbito no solo familiar, sino también comunitario.

Yo veo que hoy en día en nuestra sociedad parece que nuestros mayores son estorbos. Personas que ya no aportan nada en la vida, cuando sí aportan mucho, lo que ellos han vivido. Para que la gente joven vea que no es tan fácil la vida, para que vean la gente joven que la vida hay que currársela $\left(\mathrm{Em} \downarrow{ }_{7} 89_{23}\right)$

\section{Figura 2}

\section{Imagen social de la vejez}

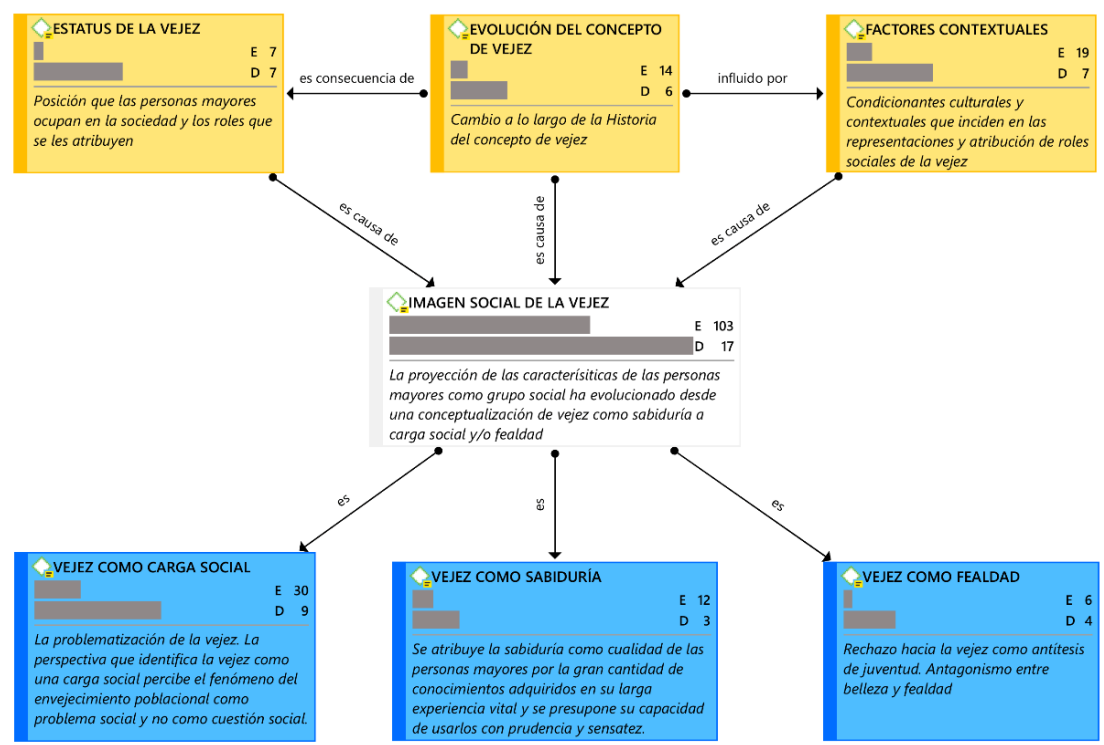

Fuente: elaboración propia 


\section{REFLEXIONAR PARA AVANZAR: LA NECESIDAD DE RECONCEPTUALIZAR LA VEJEZ}

Los cambios demográficos y de la estructura social han transformado la extensión de las etapas del ciclo vital de forma que la vejez puede llegar a ser el periodo más largo de la vida, hecho sin precedentes y que requiere una reevaluación de las expectativas vitales. Esta nueva realidad requiere una redefinición conceptual de la vejez más fidedigna a su significación actual, así como una planificación estratégica de esta etapa, al igual que hacemos con la infancia, la juventud o la edad adulta.

Los participantes, por ejemplo, apuntan que en la actualidad la vejez es un periodo demasiado extenso para ser considerado como una etapa unitaria. En la línea de la teoría de las tres etapas de la jubilación de Reitzes y Mutran (2004), resaltan la relevancia de los Go-Go como parte del sistema productivo de las sociedades posindustriales.

Están activos. Solo se han jubilado y están en esta etapa. Pueden estar en el gimnasio poniéndose en forma como diciendo «todavía puedo, yo puedo»o apuntándose a todas las agencias de viajes recorriendo el mundo o a lo mejor en su casa limpiando como locos, porque tienen todo el tiempo del mundo para hacer lo que quieran. Están como en una fase de querer hacerlo todo, muy activos (Ae19)

Para el mercado, estos mayores con buenos niveles de salud, tiempo disponible, estilos de vida activos y capacidad adquisitiva son potenciales clientes que, hasta hace poco, no habían sido considerados objetivo comercial. Surge de ahí un nuevo nicho de mercado como evidencia la proliferación de anuncios publicitarios de productos diseñados especialmente para personas mayores.

A los que están en forma, las empresas y los medios intentan venderles productos, coches, tabaco. Son un objetivo. Los mayores que van al McDonald. Las empresas hacen cosas que puedan vender para los Go-Go. Productos que cuando los vean no digan «iAy, eso es para jóvenes!». No, cosas que sean para los mayores también. Hacen un montón de productos para las personas mayores en sus estrategias (...) porque la gente mayor tiene tiempo y dinero (Ae7)

También los Go-go son actores valiosos que ocupan posiciones estratégicas en los procesos de toma de decisiones de las empresas, con cargos honoríficos en los consejos de dirección, que ponen en valor su experiencia vital. Es de 
resaltar que esta referencia solo se circunscribe al género masculino y a las clases más altas con capacidad de influencia previa, evidenciándose la desigualdad que enfrentan las mujeres también en la vejez.

Y también, en la vida pública, en la política, en los medios, hay muchos, especialmente hombres, en posiciones importantes en organizaciones, que están en los $70 \mathrm{o} 80$, porque tienen un montón de experiencia $\left(\mathrm{Ae}_{7}\right)$

Sin embargo, en las otras dos etapas, cuando aparecen las limitaciones funcionales, el espacio público queda restringido. En línea con Marshall (2012), se evidencia que el envejecimiento produce la invisibilización del individuo tanto en el ámbito personal como social.

Creo que es difícil para la sociedad si tú no ves a las personas mayores, cuando están totalmente alejados. No los ves, no puedes pensar en sus problemas (Ae19)

A este respecto, consideramos necesario redimensionar la conceptualización de la vejez incorporando otros elementos más allá de la reducción de capacidades funcionales o la pérdida del rol laboral. En línea con Sandberg (2013), planteamos afrontar el análisis de la vejez desde un enfoque de envejecimiento afirmativo que incluya la heterogeneidad creciente de las personas mayores como grupo poblacional, con mayor nivel educativo, estilos de vida dinámicos y más oportunidades. En este sentido, proponemos la búsqueda de la seneficiencia como enfoque del envejecimiento centrado en un buen envejecer tanto individual, proporcionando oportunidades para un envejecimiento exitoso, como social, planificando y canalizando estratégicamente las aportaciones sociales durante la vejez para un envejecimiento prosocial. Esta perspectiva, más amplia e integradora, permitiría incluir a aquellas personas que no se autoidentifican como mayores, cuestión que resulta clave para avanzar en la ruptura de estereotipos heteronormativos, prejuicios y discriminación, así como para lograr una representación social más fiel de la vejez en el escenario actual y para el diseño de estrategias de participación activa exitosas. 
Figura 3

\section{La vejez como el periodo más largo de la vida: 3 etapas}

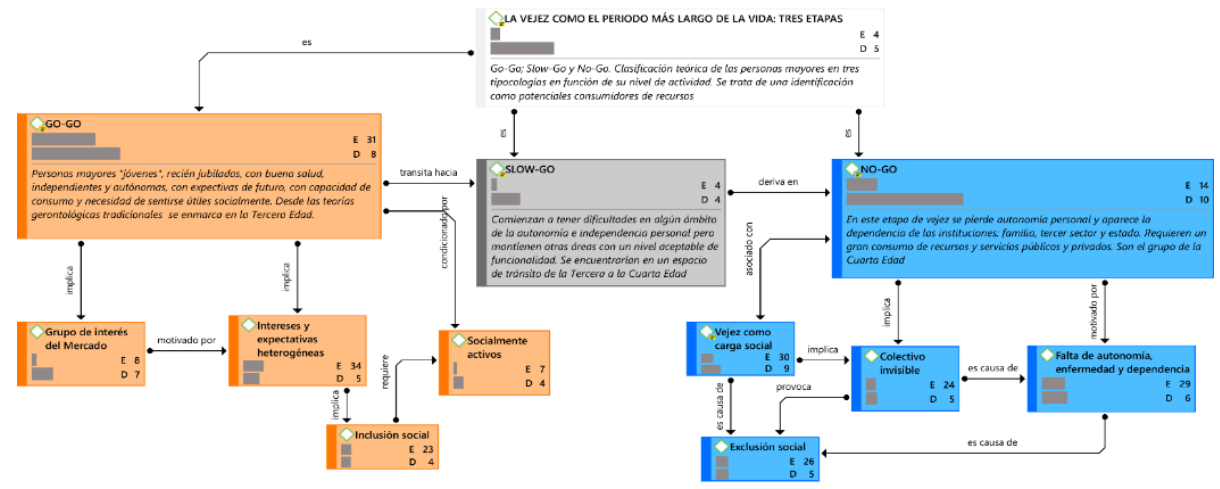

Fuente: elaboración propia

Figura 4

Necesidad de reconceptualizar la vejez

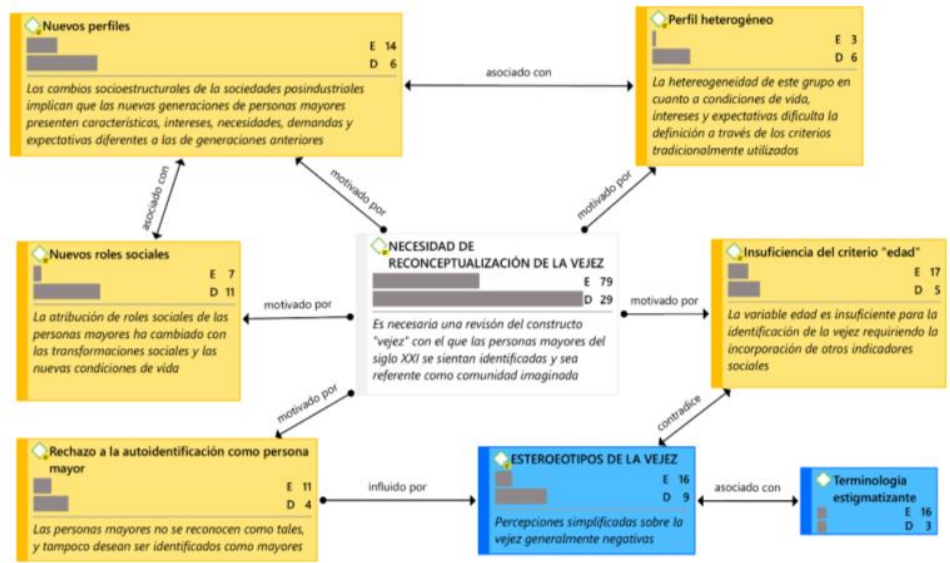

Fuente: elaboración propia 


\section{CONCLUSIONES}

Del presente estudio se deduce que los estereotipos de vejez existentes no se adecuan a las percepciones que de sí mismas tienen las personas mayores. Así, los hallazgos revelan la apreciación de pérdida de reconocimiento social hacia las personas mayores y la consolidación de una imagen social negativa conformada por prejuicios y estereotipos. Entre estos elementos asociados a la vejez en el imaginario colectivo los informantes destacan: la fealdad de lo viejo como algo no deseable, la improductividad social, la imputación de carga social y el sentimiento de culpabilidad de la vejez. Por otra parte, si bien coincidiendo con estudios previos (Fernández-Ballesteros, Schettini, Santacreu y Molina, 2012), no se evidencian diferencias culturales en la definición del buen envejecer: en el contexto español se observa una mayor prevalencia de una concepción de la vejez estereotipada negativamente asociada con falta de salud, dependencia, inactividad, falta de sentido vital, improductividad, inutilidad y carga social.

Estas percepciones producen un fuerte rechazo a la autoidentificación social de la vejez, no siendo esta percibida como una comunidad imaginada deseable. Las personas mayores no se reconocen en los estereotipos de vejez ni encuentran elementos de reconocimiento grupal. Por otra parte, los rituales de reafirmación asociados a la vejez como la jubilación son casi siempre definidos como pérdida de valor social, no ofreciendo por tanto consistencia para la consolidación de una identidad social.

Sin embargo, las circunstancias sociales actuales de las personas mayores no reproducen los escenarios tradicionales de la vejez ni sus expectativas encuentran respuesta en los recursos que la sociedad ofrece. Por otra parte, las transformaciones sociales en las estructuras laborales y los modelos de familia han cambiado el rol social de las personas mayores, asumiendo frecuentemente nuevas responsabilidades en la esfera privada y pública.

No es de extrañar, por tanto, que las personas mayores de la sociedad posindustrial no se autoidentifiquen como mayores, dado que sus experiencias, vivencias, estilos de vida y, sobre todo, sus expectativas no se adecuan al modelo de cualidades y de conducta tradicionalmente asociados con el estatus de persona mayor. Los mayores del siglo XXI gozan de buena salud, son autónomos $\mathrm{y}$ activos, tienen responsabilidades y expectativas sobre el futuro $\mathrm{y}$, en muchas ocasiones, son un activo importante en el sostenimiento de la familia y la co- 
munidad. En este sentido, se evidencian diferentes autopercepciones y valoraciones culturales del rol social de la vejez. Así, las personas mayores españolas, aunque identifican la importancia de su rol en las tareas de cuidado en el ámbito familiar, no lo valoran como aportación a la comunidad. Los mayores alemanes, en cambio, subrayan estas aportaciones privadas, así como otras en el ámbito comunitario (voluntariado, etc.), reivindicando el reconocimiento de su valor social.

En relación con esto, consideran que ser mayor no depende de la edad, sino de la capacidad, concibiendo la vejez como la falta de capacidad para desarrollar funcionamientos válidos personal y socialmente. Se valora, por tanto, la autonomía, que engloba tanto la capacidad para tomar decisiones personales como la capacidad de realizar acciones.

Se pone de manifiesto la necesidad de redefinir el concepto de vejez abordándose las nuevas dimensiones sociales asociadas al fenómeno y clarificar otros conceptos asociados para una mayor precisión terminológica. Para ello resulta imprescindible la incorporación no solo de criterios de edad, sino de otros indicadores de vejez (entre los que destacamos la percepción subjetiva de bienestar), así como un cambio de perspectiva que atienda la heterogeneidad creciente de las experiencias, las vivencias y las expectativas de las personas mayores y el nuevo perfil de mayores de las sociedades posindustriales. A este respecto, proponemos la seneficiencia como modelo de un buen envejecer individual y social. Consideramos que las transferencias sociales de este tipo de enfoques de afrontamiento facilitarán la extensión de estereotipos positivos sobre la vejez que estimulen el orgullo de envejecer o elder pride. Por último, es necesario superar la visión heteronormativa de la vejez, incluyéndose análisis relativos a las distintas realidades socioculturales presentes en los distintos contextos y que condicionan la concepción y las atribuciones sociales a la vejez, así como la percepción subjetiva de bienestar ligada al envejecimiento exitoso. Asumiendo las limitaciones del presente trabajo con respecto a la representatividad de la muestra, se plantea la necesidad de incluir, en futuros estudios, una muestra más heterogénea de personas mayores que permita establecer análisis diferenciales según las circunstancias de partida y, por ende, una mayor generalización de los resultados. Asimismo, resulta fundamental realizar un análisis de género en profundidad sobre el tema en cuestión. 


\section{BIBLIOGRAFÍA}

Amezcua, Teresa y Alberich, Tomás (2017). Los nuevos roles de las personas mayores en el hogar y la sociedad: ser mayor ya no es lo que era. Paraninfo Digital, 26(2). Recuperado de: http://www.indexf.com/para/n26/o01.php

Amezcua, Teresa y García-Domingo, Marta (2020). Principales problemáticas e inquietudes de las personas mayores en las sociedades posindustriales. Análisis cualitativo en los contextos español y alemán. Methaodos.revista de ciencias sociales, 8(2), 271-287. doi: http://dx.doi.org/10.17502/mrcs.v8i2.418

Amico, Lucía (2009). Envejecer en el siglo XXI. No siempre querer es poder. Hacia la deconstrucción de mitos y la superación de estereotipos en torno a los adultos mayores en sociedad. Recuperado de: http://margen.org/suscri/margen55/ amico.pdf [Consultado el 22 de julio de 2020].

Andrews, Molly (1999). The seduction of agelessness. Ageing \& Society, 19(3), 301-318. doi: https://doi.org/10.1017/So144686X99007369

Barrett, Anne, Redmond, Rebeca y Von Rohr, Carmen (2012). Avoiding aging? Social psychology's treatment of age. The American Sociologist, $43(3), 328-347$.

Butler, Robert (1975). Why Survive? Being Old in America. New York: Harper \& Row.

Butler, Robert (1980). Ageism: A foreword. Journal of Social Issues, 36(2), 8-11.

Cantos, Mercedes (2019). Hacia otra forma de envejecer: estereotipos y realidades de la vejez en España. Tesis doctoral. Madrid: Universidad Complutense de Madrid.

Clavan, Silvia (1978). The impact of social class and social trends on the role of grandparent. The Family Coordinator, 27(4), 351-357. doi: https://doi.org/10.2307/583437

Cook, Ian Gillespie y Halsall, Jaime (2012). Aging in comparative perspective: Processes and Policies. New York: Springer. doi: https://doi.org/10.1007/978-1-4614-1978-5 
De Beauvoir, Simone (1970). Old Age. Middlesex: Penguin Books.

De Jouvenel, Hugues (1989). Europe's ageing population: trends and challenges to 2025. Paris: Futurible International.

Esteban, Luz (2004). Antropología del cuerpo: género, itinerarios corporales, identidad y cambio. Barcelona: Bellaterra

Fernández-Ballesteros, Rocío, Schettini, Rocío, Santacreu, Marta y Molina, Mari Ángeles (2012). Lay Concept of Aging Well According to Age: A ReAnalysis. Journal of the American Geriatrics Society, 6o(11), 2172-2173. doi: https://doi.org/10.1111/j.1532-5415.2012.04232.x

Fernández, José Manuel y Kehl, Susana (2001). La construcción social de la vejez. Cuadernos de Trabajo Social, 14, 125-161.

Fries, James (1980). Aging, natural death, and the compression of morbidity. New England Journal of Medicine, 303(3), 130-135. doi: https://doi.org/10.1056/NEJM198007173030304

García, Begoña y Alfageme, Alfredo (2012). Curso vital: viejas estructuras y nuevos retos. Recerca. Revista de Pensament i Análisi, 9, 7-12. Recuperado de: https://www.e-revistes.uji.es/index.php/recerca/article /view/144

García-Domingo, Marta (2019). Reflexión profesional sobre la realidad de la intervención social: retrocesos propios de un periodo de postcrisis y propuestas de mejora. Alternativas. Cuadernos de Trabajo Social, 26, 103-122. doi: https://doi.org/10.14198/ALTERN2019.26.05

García-Domingo, Marta y Sotomayor, Eva (2017). El rol del profesional del Trabajo Social en una coyuntura de crisis: oportunidades de la adaptación a un contexto cambiante. Trabajo Social Global, 7(12), 4768. Recuperado de: http://hdl.handle.net/10481/47010

García-Rodríguez, Beatriz (2007). Bienestar subjetivo y felicidad en la vejez. En Ballesteros, Soledad (ed.). Envejecimiento saludable: Aspectos biológicos, psicológicos y sociales (273-308). Madrid: Universitas. Recuperado de: https://dialnet.unirioja.es/servlet/libro?codigo $=570341$

Higgs, Paul y Gilleard, Chris (2021). Fourth Ageism: Real and Imaginary Old Age. Societies 2021, 11, 1-7. doi: http://dx.doi.org/10.3390/soc11010 012 
Gott, Merryn (2004). Sexuality, sexual health and ageing. UK: McGrawHill Education.

Gullette, Margaret Morganroth (1997). Declining to decline: Cultural combat and the politics of the midlife. Charlottesville: University of Virginia.

Gutiérrez, Marta y Hernández, Daniel (2013). Las relaciones intergeneracionales en la sociedad actual: un imperativo necesario. Educación Social. Revista de Intervención Socioeducativa, 55, 135-145.

Johnson, Colleen y Barer, Barbara. (1997). Life beyond 85 years: The aura of survivorship. San Francisco: Springer Publishing Co.

Kahana, Eva y Kahana, Boaz (1996). Conceptual and empirical advances in understanding aging well through proactive adaptation. En Bengtson, Ven L. (ed.) Adulthood and aging, research on continuities and discontinuities (18-40). New York: Springer.

Katz, Jeanne, Holland, Caroline y Peace, Sheila (2013). Hearing the voices of people with high support needs. Journal of Aging Studies, 27(1), 52-6o. doi: https://doi.org/10.1016/j.jaging.2012.11.003

Márquez-González, María (2008). Emociones y envejecimiento. Informes Portal Mayores, 84. Recuperado de: https://bit.ly/2SkSQIk [Consultado el 18 de septiembre de 2020].

Marshall, Leni (2012). Through (with) the Looking Glass: Revisiting Lacan and Woodward in "Méconnaissance," the Mirror Stage of Old Age. Feminist Formations, 24(2), 52-76. doi: https://doi.org/10.1353/ff.2 012.0022

Medina, Raquel (2018). Vejez, envejecimiento y edadismo. En Rodríguez, María y Aguado, Txetxu (eds.). Representaciones artísticas y sociales del envejecimiento (27-41). Madrid: Dykinson. doi: https://doi.org/10.2307/j.ctv301f4f.4

Navarro, Ana Belén, Bueno, Belén, Buz, José y Mayoral, Paula (2006). Percepción de autoeficacia en el afrontamiento de los problemas y su contribución en la satisfacción vital de las personas mayores. Revista Española de Geriatría y Gerontología, 41(4), 222-227. doi: https://doi.org/10.1016/So211-139X(o6)72959-6 
Nelson, Todd D. (2005). Ageism: Prejudice against our feared future self. Journal of social issues, 61(2), 207-221. doi: https://doi.org/10.1111/j.15404560.2005.00402.x

Neugarten, Berenice (1974). Age Groups in American Society and the Rise of the Young-Old. The ANNALS of the American Academy of Political and Social Science, 415(1), 187-198. doi: https://doi.org/10.1177/000271627441500114

Pizzorno, Alessandro (1978). Political exchange and collective identity in industrial conflict. En Crouch, Colin y Pizzorno, Alessandro (eds.). The resurgence of class conflict in Western Europe since 1968 (277298). London: Palgrave Macmillan

Reitzes, Donald y Mutran, Elisabeth (2004). The transition to retirement: Stages and factors that influence retirement adjustment. The International Journal of Aging and Human Development, 59(1), 63-84. doi: https://doi.org/10.7440/res42.2012.03

Ribera, José Manuel, Bustillos, Antonio, Guerra, Ana Ilenia, Huici, Carmen y Fernández-Ballesteros, Rocío (2016). ¿Se discrimina a los mayores en función de su edad? Visión del profesional. Revista Espanola de Geriatría y Gerontología, 51(5), 270-275. doi: https://doi.org/10.1016/j.regg.2016.03.005

Riley, Matilda White y Riley, John W. Jr. (1994). Structural lag: Past and future. En Riley, Matilda White, Kahn, Robert y Foner, Anne (eds.). Age and structural lag: Society's failure to provide meaningful opportunities in work, family, and leisure (15-36). New York: John Wiley \& Sons

Rowe, John W. y Kahn, Robert L. (1997). Successful aging. The gerontologist, $37(4)$, 433-440. doi: https://doi.org/10.1093/geront/37.4.433

Sandberg, Linn (2013). Affirmative old age: The ageing body and feminist theories on difference. International Journal of Ageing and Later Life, 8(1), 11-40. doi: https://doi.org/10.3384/ijal.1652-8670.12197

Sapir, Andre (2006). Globalization and the reform of European social models. Journal of Common Market Studies, 44(2), 369-390. doi: https://doi.org/10.1111/j.1468-5965.2006.00627.x 